\title{
Image-text Relation in Modern Children's Picture Book Design
}

\author{
Kun Tong \\ College of Fine Arts \\ Huanggang Normal University \\ Huanggang, China 438000
}

\begin{abstract}
Image-text relation is the soul of modern children's picture book design and directly influences impressions and reading experience of readers. With the development of society, text gradually evolves from a kind of graphic symbol to a tool of paraphrasing. Images develop from cultural appurtenance into independent artistic form. Since the modernism, "individualization", "diversification" and "internationalization" have become main characteristics of modern art. Image and text show new relations. The artistry and speaking right of image improve obviously. The interpretation function of text shows greater flexibility. Children's picture book serves as the book form with the largest amount of distribution and the most unique audiences. Image and text show the most essential attributes here. They keep independent as well as influence each other and bring out the best in each other.
\end{abstract}

Keywords-modern; children's picture book; image-text relation

\section{STRENGTH OF GRAPHIC LANGUAGE}

Using figures to arouse sensibility derives from the New Wood-cut Movement in early stage of the 20th century. Meanwhile, it is also the origin of modern children's picture book in China. The emergence of New Wood-cut Movement has three reasons: social revolution of extreme misery, rise of expressionism and illiteracy of a large number of unprivileged people. Lu Xun looks forward to make the domestic strife and foreign aggression of our country and long-standing abuse of spirit public through an effective way, in order to arouse introspection and self-consciousness of most people and promote the success of democratic revolution. The "New Wood-cut Movement" rises in response to the proper time and conditions. The key is to establish a kind of graphic language that suits both refined and popular tastes and everyone knows about it. It shall achieve the effect of shocking by the sight and express the perceptual intuition and profound meaning that is difficult for literal expression to realize. That kind of picture is concise, sharp, and appropriate and can go straight to the heart of the matter. Lu Xun thinks that "when revolution begins, the application of wood block is the most extensive. Although it is extremely hurried, it can be done in a moment."[1] Under the

Youth fund project of humanities and social sciences general project of Ministry of Education in 2015: Modernism of Children-Research on Idea of Modeling and Method of Modern Children's Picture Book. Project grant no.: 15YJC760086. This article is the research achievement in medium-term of this project.

Youth project of humanities and social sciences of Education Department in Hubei province in 2014: the conclusion result of research on idea of modeling and method of modern children's picture book. Project no.: 14Q113 advocacy of Lu Xun, printmakers such as Liu Xian, Gu Yuan and Zhao Yannian create a host of excellent wood block works that enjoy popular support "Fig. 1". It not only plays the role in publicizing the situation, but also promotes the development of modern painting of our country. At the same time, it proves that it is feasible and effective to use the power of pictures to carry out the revolution.

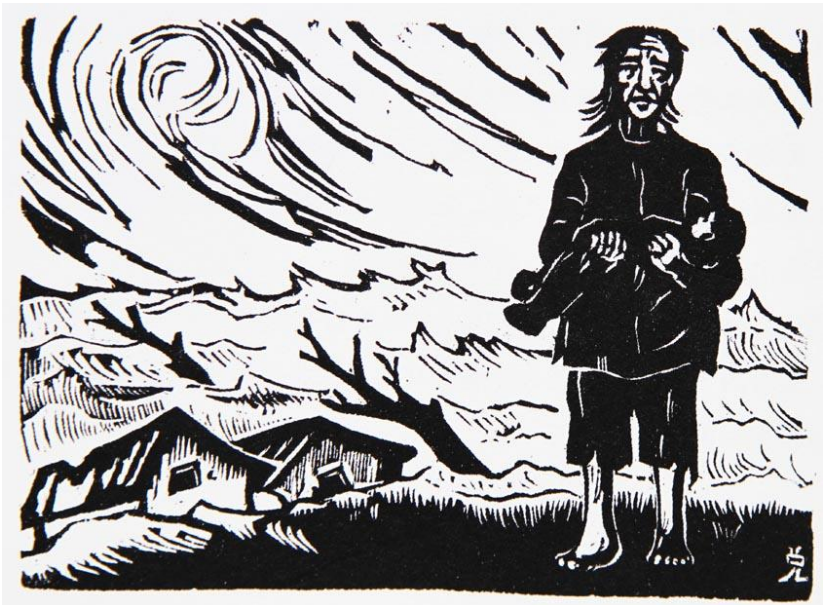

Fig. 1. Liu Yan, One of the Stories without Word, 1937

The education in the Republic of China thinks that the biggest function of education is to make people produce sensitivity. At that time, "the conquest of Qing dynasty makes most of the Chinese people surrender as slaves for a long time. The human relation of the emperor and subjects is demoted to master and servant! The trample of this spirit make cultural elite of China lose innovation power at Qing dynasty."[2] Looking back to see textbooks in the Republic of China era, most of them adopt the form of image-text combination. Illustrations in the Republic of China often do not try to please the public with claptrap. The stroke is clear and it furthest simplifies great truth. People without distinction of sex and age can read vivid new meaning and true feelings from the pictures. It embodies the effect that uses pictures to benefit words. As Mr. Feng Zikai said, "Using the way of comfort to influence our feelings imperceptibly is the great use of painting."'[3]

From totem worship in the Old Stone Age to political practice that uses religious symbols to perform imperial autocracy in mediaeval times and modern times; from 
generation of expressionism to wide spread of cubism, abstractionism and pop art, all of them prove the feasibility and enormous energy of graphic language in publicizing artistic ideas and social concept. W.J.T. Michelle, an American image theorist, thinks the relation between image and text is a kind of power relation in reality, as well as the relation between watching and reading. They are not only two kinds of different modes of reappearance, but also express cultural value with deep contradiction. He thinks that since the contemporary age, "image (picture)" has turned from reappearance to a kind of complex and diversified cultural interpretation. Visual experience (pictures) can not be explained by text completely. Watching is profound, so is reading. In modern society, as a kind of irreplaceable communication media, graphic language often can release more profound meaning of more levels and types. The pictures in children's picture book are typical forms of graphic language. It has great power to influence the mind and enlighten the wisdom "Fig. 2" .

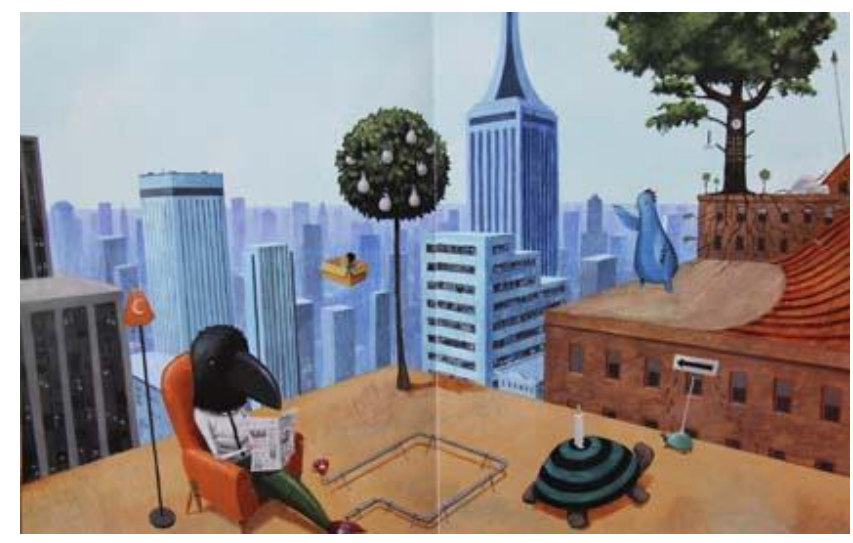

Fig. 2. (Denmark) Solon Jason, Flying Box, 2008

\section{USE PICTURES TO GET THE UPPER HAND}

In a broad sense, picture book derives from the requirements for cognition and aesthetics, which is similar to the origin of art of painting. With the development of society, picture books become a kind of practical cultural carrier because of its growing functionality. One of the contributions of modernism is that it recovers artistic attribute of picture book and make the drawing of pictures in picture book become independent artistic creation. All kinds of painting, media and language can be used by the creation of picture book, in order to present cozy and magnificent watching and reading experience.

Since the 20th century, the art of painting and development of picture books contrast finely with each other. The concept of modernism adds new vitality to the picture book design. The greatest painters in the 20th century such as French painter Pierre Bonar, who is good at subjective color expression and writing brush stroke, British painter Lucian Freud, who is famous for representational realism and rational thinking, British painter David Hockney, who takes new realistic modeling and bright-colored color configuration as style label, the great Spanish painter Picasso and Andy Warhol, the representative personage of American pop art, have the experience of drawing illustration for books. In our country, there are precedents that famous painters such as Feng Zikai, Cheng Shifa and Ye Qianyu draw illustration for children's books. Their creation makes books become more perfect. Besides, these pictures are rare artistic works as well as the most important part of picture books.

First of all, the pictures on excellent children's picture books shall conform to children's mind and the ability of visual judgment and become memorable and impressive for children. Children are good at concluding essential impression of things, which is concise as well as the most intrinsic. Painters of children's picture books need to more intuitively express the "nature" of children's understanding by using concise lines and shapes. In addition, they should be inclined to suppress their own skills, in order to make them simple and rough. Complex modeling and skill will make children produce sense of distance and awe. That result runs counter to the original intention of modernism. Children's particularity on physical growth and cognitive level decides that they tend to accept obvious lines and shapes. It can meet their requirements for description as well as cultivate their confidence in expression.

Pictures on children's picture books have the ability of independent expression instead of depending on text. Pictures on picture books are not trying to tell children a story, but guiding them to form independent visual perception and psychological experience. Sometimes children can see many stories in one picture, which also conforms to picture theory of W.J.T Michelle. Tadashi Matsui, the Japanese painter for picture book, even thinks that "stories created by children are the real picture book as well as real experience of picture book." 4 In reality, children are not only acceptors of picture book but also participants and creators. Picture books provide a path for works interpretation. Children often can create new paths from a lot of visual information of the picture, which have more embodiments on preschool children. On one hand, preschool children have weak literacy and rely on less written message and they are more accustomed to seeking and finding from pictures. On the other hand, preschool children have limited cognition for concepts and can unconsciously avoid the situation that concepts leave the strongest first impressions, keep strange feeling for logical relationship of visual information, then get a personalized reading mode for picture books.

Pictures have the function guided by modern aesthetics. Picture books are created in limited space but they can cover the entire modeling arts. The core of modern modeling concept is unique rendering of visual sense and thinking as well as views and actions of painters on objectivity and subjectivity. Because "individuation" occupies important position in modern art and internationalization trend of art has deepened since the 20th century, it makes modernist painting present very complicated situation. In the face of such situation, the design of pictures on picture book can only selectively accept achievements of modern art. The first is to make children produce sensitivity and sympathy. The second is to have meaningful forms, which are the embodiments of aesthetic spirits. The last is to embody independent thinking of painters, which is the performance of individuation. Modern children's 
picture books use pictures to guide children to perceive beauty, realize beauty, discover beauty as well as create beauty.

Pictures have the function to guide children's mind to become perfect. Philosopher John Dewey thinks that aesthetic experience has the ability to influence morality. In the "Picture Learning Movement" in the 1920s, it widely spread this kind of belief in class in America: The power of art can bring the improvement of people and culture. Children are special audiences and the "people" remained to improve. At the same time, they are power of preparation for the development of times. In the process of children education, children's picture books have the function and responsibility of guidance and education. Compared with text, images are more visualized and include more diversified and extensive information. It has faster and more accurate characteristics on spread of ideas. At the beginning of the 20th century, Mr. Feng Zikai created Paintings on the Preservation of Life, using the form of excellent picture and its accompanying essay to show our daily life. It seems to describe the harmony between human and nature. In reality, his real intention is to arouse the awakening of human nature, from numb to sensitive, from cold to tender, in order to advocate universal morality and responsibility and finally make people establish scientific and democratic world outlook and become independent, kind-hearted and strong people "Fig. 3". Something of success of this works lies in that Mr. Feng Zikai keeps a foothold on reality, uses the most concise lines to create the most vivid image, uses the most simple picture to express the most profound theme, describes manifold text in simple strokes and creates a pattern of manifestation that suits both refined and popular tastes and explains the profound in simple terms for pictures in picture books. Feng Zikai thinks that children are the most sensitive and sensitivity is the source of beauty and art. Therefore, children's perception is also the state that is closest to art. The stroke of Feng Zikai gets close to children's perception. It is intended to touch human nature. Meanwhile, it also conforms to the nature of modeling concept in modern times.

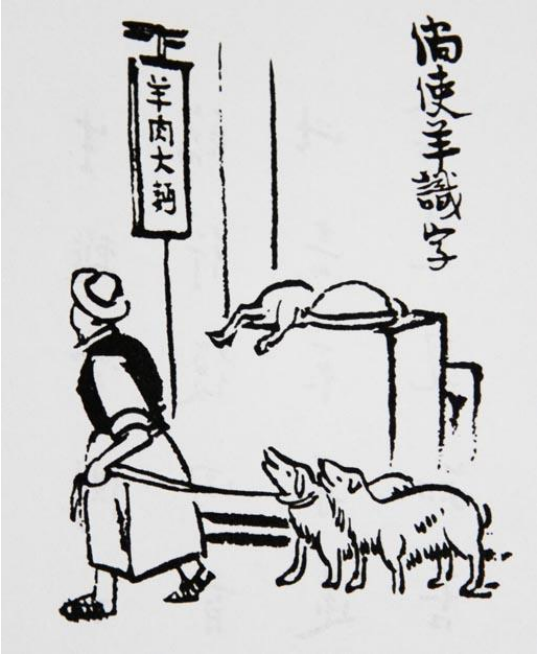

Fig. 3. The first episode of Paintings on the Preservation of Life, Feng Zikai: If Make Sheep Learn to Read, 1929

\section{CONCEALING MistaKeS AND ERRORS WITH BEAUTIFUL} WORDS

The text design of picture books strives to go through the motions. Excessive interpretation of text will restrict children's thinking as well as reduce persuasion and artistry of pictures.

Since its appearance, text has always undertaken the tasks of explanation and expression. It originates from the requirements of cognition. It is the symbolized visual mark. Looking from the development history of art, text should be the form of artistic expression that is most widely used and enjoys the most popular support. In order to meet the requirements of efficiency and accuracy, text always separates from images and becomes independent visual symbol that is easier to spread. But as a kind of trace, text still has general characteristic of modeling art. Ms. Cecilia Lindqvist, a sinologist in Sweden starts with the modeling features of Chinese characters and systematically researches origin and development of Chinese characters. The basic viewpoint of Kingdom of Chinese Characters written by her is that text comes from requirements of life and production. They go through the period of images before they become the real Chinese characters.

Today, the mission of images is redefined. The function of reappearance of painting is gradually shrunk. As a kind of inefficient, elaborate and complicated creation activity, figurative realistic painting receives severe challenges. Paintings once created for court and aristocracy or hall and temple gradually move toward the market and become decoration in home of ordinary people. The revolution of modern image technology blurs the boundary between serious art and pop art. Images that can be produced in a moment and spread widely appear overwhelmingly. "Everyone is artist" is the most dramatic and truest portrayal in image era. Images walk into our life, just as text breaks away from pictures and walks toward books. Images gradually surpass text and have extraordinary flow characteristics-efficient, extensive and profound. People can more profoundly and effectively master information through interpreting drawings. Just as Lu Xun said in a letter written to young printmaker Liu Xian about differences between old and new woodcut New Year paintings in 1934, "For the old one, we know the story first and then watch the painting; for the new one, we have to watch the painting first and then know the story." 5 On the basis of meeting conveying function of general information, combine with impressive artistic performance, so that we can make pictures produce warm and unique aesthetic feeling immediately.

Making a general survey of development of children's picture books, it is easy to find that the area occupied by pictures in picture book becomes increasingly large and text occupies smaller and smaller area. The pictures are more delicate and have artistry. The text is more concise and has flexibility. Text gradually quits the function of explanation and plays the function of guidance. Pictures on children's picture books absorb concept and method of modern modeling art and avoid contradictions between image duplicating and personalized performance. Both realistic portrayal and abstract and performance take children's cognitive ability and 
characteristics as the standard, take positive factor of modern aesthetics as the guidance, creating excellent works that sparkle with intelligence and shock our heart "Fig. 4".

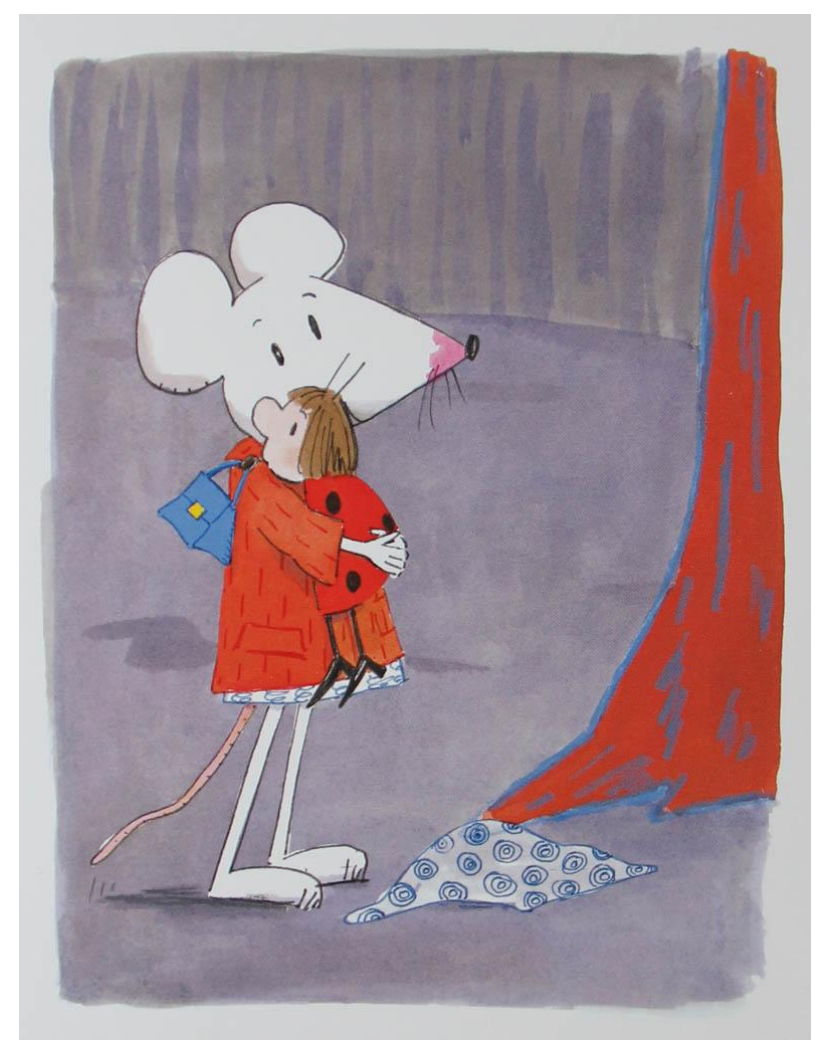

Fig. 4. (France) Catalina Walkers, Small House of Duoduo and Meimei,

\section{CONCLUSION}

It is the principle of modern children's picture book design for image and text "complement each other". Excellent children litterateur should understand the drawing. Excellent painter for picture book shall have very high literary accomplishment. Sometimes they are a person. Sometimes they strive for a common goal-arouse the feeling of children of sensitivity, sympathy and exploration. Image and text jointly form the world of picture book. In modern society with high-speed expansion of information, children's picture book gradually returns the nature of "picture book"-use image to pass beauty and thoughts. Higher requirements are put forward for artistry of children's picture book design.

Text serves as the secondary part in children's picture book. It doesn't mean neglecting the importance of text. On the contrary, it puts forward higher requirements for text. The more concise the text, the higher degree of summary it will be. Besides, it can cause infinite association.

\section{REFERENCES}

[1] Wang Renyan. Print Pioneer Liu Xian.China Water Power Press, 2009, p. 15.

[2] Xu Zhuoyun. We and Others. Sanlian Bookstore, 2015, p.120.
[3] Feng Zikai. Interest of Art, Hunan Literature and Art Publishing House, 2002, p. 34.

[4] [Japan] Kunio Yanagida, Tadashi Matsui, Hayao Kawai, translated by Zhu Ziqiang. Ehon No Chikara. Guizhou People's Fine Arts Publishing House, 2011, p. 108.

[5] Wang Renyan. Print Pioneer Liu Xian.China Water Power Press, 2009, p.30. 